Published in final edited form as:

Curr Treat Options Oncol. 2015 January ; 16(1): 317. doi:10.1007/s11864-014-0317-1.

\title{
Immunotherapy for Ovarian Cancer
}

Justin M. Drerup, B.S. ${ }^{1,2}$, Yang Liu' ${ }^{2,3}$, Alvaro S. Padron², Kruthi Murthy², Vincent Hurez², Bin Zhang ${ }^{4}$, and Tyler J. Curiel, M.D., M.P.H. ${ }^{1,2,5}$

${ }^{1}$ Department of Cellular and Structural Biology, School of Medicine, University of Texas Health

Science Center, San Antonio, TX 78229 USA

${ }^{2}$ Department of Medicine, School of Medicine, University of Texas Health Science Center, San Antonio, TX 78229 USA

${ }^{3}$ Department of Clinical Medicine, Xiangya School of Medicine, Central South University, Changsha, Hunan 410013, P.R. China

${ }^{4}$ Robert H. Lurie Comprehensive Cancer Center, Department of Medicine-Division of Hematology/Oncology, Northwestern University Feinberg School of Medicine, Chicago, IL 60611, USA

${ }^{5}$ Cancer Therapy \& Research Center, University of Texas Health Science Center, San Antonio, TX 78229 USA

\section{Keywords}

ovarian cancer; immune therapy; cancer vaccine; monoclonal antibody; early phase clinical trials; oncolytic virus; radioimmunotherapy; CAR T cell; adoptive immunotherapy

\section{Introduction}

Ovarian cancer (OC) is considered to arise from epithelial cells encapsulating ovaries, stromal cells, or ova, although recent evidence suggests origins in Fallopian tubes and other sites as well ${ }^{[1]}$. The great majority of OC are epithelial carcinomas and often present with advanced or metastatic disease. Although chemotherapy and surgical debulking can eliminate clinically apparent cancer, patients often succumb to chemotherapy-resistant tumor relapse within several years after initial remission. Immunotherapy for OC could be effective $^{[2-9]}$ as OC cells express immunogenic tumor-associated antigens that elicit detectable, specific immune responses ${ }^{[10-19]}$. The positive correlation between $\mathrm{OC}$ survival

\footnotetext{
Corresponding author: Tyler Curiel, M.D., M.P.H., Adult Cancer Program, 8403 Floyd Curl Drive MC8252, San Antonio, TX 78229, curielt@uthscsa.edu.

Contact info:

Justin Drerup, Department of Cellular and Structural Biology, 8403 Floyd Curl Drive MC8252, San Antonio, TX 78229,

drerup@livemail.uthscsa.edu

Tyler Curiel, Adult Cancer Program, 8403 Floyd Curl Drive MC8252, San Antonio, TX 78229, curielt@uthscsa.edu

Compliance with Ethics Guidelines

Human and Animal Rights and Informed Consent This article does not contain any primary animal or human studies.

Conflict of interest

Justin M. Drerup, Yang Liu, Alvaro Padron, Vincent Hurez, and Bin Zhang declare no conflict of interest. Tyler J. Curiel has received research support and consulting fees from Eisai.
} 
and tumor infiltration with $\mathrm{CD} 8^{+} \mathrm{T}$ cells is compelling evidence that anti-tumor immune surveillance is a critical dictate of clinical outcomes in $\mathrm{OC}^{[20]}$. Despite abundant evidence that anti-tumor immunity in OC could be effective, immune-based OC therapies have generally been only modestly successful, at best. The first immunotherapy for OC used intraperitoneal injections of anti-human milk fat globulin-1 antibodies in $1987^{[21]}$, which was also among the very first uses of monoclonal antibodies as cancer immunotherapy. Additional antibody approaches followed, most notably with failure of the anti-CA-125 antibody oregovomab. Although there have been anecdotal reports of good clinical responses to newer immunotherapy approaches, there is no FDA-approved OC immunotherapy, as exists for other cancers. Nonetheless, recent data suggest that effective, tolerable OC immunotherapy could be developed in the near future. Recent advances in the understanding of OC immunopathogenesis, including understanding the immunopathogenic role of regulatory $\mathrm{T}$ cell, immature myeloid cells and dysfunctional immune co-signaling, help identify potentially more effective immunotherapy approaches. Combination immunotherapies appear more promising than individual immunotherapy agents, and immunotherapy could be combined with cytotoxic agents, small molecule inhibitors, radiation therapy or surgery based on rational concepts.

\section{TREATMENT}

- Standard of care treatment for advanced stage OC includes optimal surgical debulking combined with chemotherapy with a platinum plus taxane agent.

- Immunotherapies include passive cell transfers, active vaccinations, or cytokine, toxin or antibody infusions to stimulate antitumor immunity.

- Newer experimental approaches include combinations of immunoactive agents or combining immunotherapy with cytotoxic agents, small molecule inhibitors, surgery or radiation therapy.

\section{Surgery}

- Standard of care front line surgery consists of optimal tumor debulking where feasible, or debulking as much primary tumor as possible.

- Surgery is also used in recurrences and salvage settings, occasionally with curative intent, but more often for patient comfort or to preserve organ function.

- Surgical debulking as an adjunct to immunotherapy is in the exploratory phase.

\section{Interventional procedures}

\section{Radiotherapy}

- External beam irradiation is not typically front-line OC therapy, but is used to reduce surgically inaccessible tumors or for palliation.

- The efficacy of combined external beam irradiation with immunotherapy is under investigation in other cancers ${ }^{[22]}$ but has not yet been reported in OC. 


\section{Pharmacologic treatment}

Chemotherapy as immunotherapy: This review of OC immunotherapy will not detail front-line and salvage chemotherapeutic agents, which are discussed in detail elsewhere ${ }^{[23]}$. Chemotherapy can serve as an adjunct to immune therapies through the reduction of immune suppressive factors or by increasing immune surveillance. Fludarabine ${ }^{[24]}$ or cyclophosphamide ${ }^{[25]}$ can deplete immunopathogenic regulatory T cells. 5-fluorouracil can deplete cancer-promoting myeloid-derived suppressor cells in preclinical models ${ }^{[26]}$. Anthracyclines can increase the immunogenicity of tumors through the uncovering of tumor-associated antigens by tumor lysis or release of danger signals, such as high-mobility group box $1^{[27]}$. There is a clear rationale to combine certain cytotoxic agents with immune therapies.

\section{Monoclonal antibodies}

Anti-milk fat globulin-1: The first therapeutic antibodies to treat human OC were antihuman milk fat globulin-1 antibodies radiolabeled and injected into the peritoneum, reported 27 years ago[21]. Treatment responses were positively correlated with irradiation doses and inversely correlated with tumor volumes. Additional antibodies continued to highlight the relative safety of intraperitoneal antibody injections, and produced occasional long-term clinical responses[28]. However, a phase II trial of ${ }^{90}$ yttrium-labeled anti-human milk fat globulin-1 antibodies did not show significant clinical benefits in 25 patients. Further doseescalations produced myelosuppression ${ }^{[29]}$, limiting the approach.

A phase I/II trial using ${ }^{90}$ yttrium-labeled anti-human milk fat globulin-1 in 52 patients tested standard-of-care surgery plus chemotherapy at initial OC diagnosis, followed by intraperitoneal antibody ${ }^{[30]}$. Treatment was well tolerated and 21 of 52 patients had no detectable disease at the end of therapy. At 35 months median follow-up, survival was potentially better than historical controls, suggesting possible efficacy, which was corroborated by a longer-term survival analysis in $2000^{[31]}$. More recent trials of intraperitoneal ${ }^{90}$ yttrium-labeled anti- human milk fat globulin-1 suggest that whereas it can control local (intraperitoneal) disease, distant relapses could offset any overall survival benefits. Nonetheless, further study could be warranted ${ }^{[32]}$.

Farletuzumab: Folate receptor-a is overexpressed in most OCs. Farletuzumab is a humanized anti-folate receptor-a antibody thought to function not through blocking folate transport but through antibody dependent cellular cytotoxicity. Safety and activity was demonstrated in phase I and II trials at doses from $12.5-400 \mathrm{mg} / \mathrm{m}^{2}$ in OC patients in platinum-resistant relapse ${ }^{[33,34]}$. Grade $1-2$ adverse events were noted in $80 \%$ of patients, with grade 3 fatigue reported in 2 . The most common side effects were hypersensitivity, fatigue, diarrhea, and cough/dyspnea. Ultimately, farletuzumab failed to meet its endpoint of improving progression-free survival in a recent phase III trial of 1,100 platinum-resistant OC patients (http://www.eisai.com/news/news201305.html) although a post hoc analysis suggested a trend toward improved progression free survival in OC subsets, prompting additional analyses. In another trial [33], 54 OC patients received weekly farletuzumab alone or combined with carboplatin (AUC 5-6) plus paclitaxel $\left(175 \mathrm{mg} / \mathrm{m}^{2}\right)$ or docetaxel (75 $\mathrm{mg} / \mathrm{m}^{2}$ ). Cytotoxics were given every 21 days for 6 cycles, followed by weekly 
farletuzumab until progression. 28 patients with asymptomatic CA-125 relapse got farletuzumab alone and were eligible for carobplatinum/taxane plus farletuzumab if they progressed on fareltuzumab alone. 26 patients with symptomatic relapse initially got cytotoxics plus farletuzumab and 21 additional patients had cytotoxics added after initial farletuzumab. Farletuzumab alone was well-tolerated and did not augment toxicities of cytotoxics. In the 47 patients on farletuzumab plus chemotherapy, 38 (80.9\%) normalized CA-125. Complete or partial response rates were $75 \%$ with farletuzumab plus cytotoxics. Thus, farletuzumab alone might be poorly effective, but combination with carboplatin plus a taxane could merit additional consideration in platinum sensitive first relapse.

Catumaxomab: Catumaxomab is a trifunctional antibody that kills EpCAM-expressing tumor cells, the primary cause of malignant ascites. It is approved to treat malignant ascites in Europe but not the United States. It is administered as four 3-h intraperitoneal infusions. One case report describes complete remission in an OC patient that received 4 infusions of catumaxomab alone. The most frequent adverse effects are fever, nausea, vomiting and abdominal pain. In a phase IIIb study, $25 \mathrm{mg}$ predinosolone reduced catumaxomab-related adverse events in OC patients receiving it for malignant ascites. There were non-significant trends for prednisolone to reduce time between paracenteses and for catumoxamab alone to increase overall survival, but the main finding was that prednisolone did not reduce catumaxomab-related adverse events ${ }^{[35]}$.

Ipilimumab: Immune checkpoint blockade with antibodies is emerging as potentially effective immunotherapy in many cancers ${ }^{[36]}$. Ipilimumab and tremelimumab are fully human $\operatorname{IgG1}$ or IgG2 antibodies, respectively, that antagonize the CTLA-4 immune checkpoint. Ipilimumab is FDA-approved to treat metastatic or unresectable melanoma and is the first standard-of-care immune checkpoint inhibitor. Anecdotal reports of OC responses to ipilimumab and pre-clinical findings prompted an ongoing phase II trial of ipilimumab for platinum-resistant OC (NCT01611558). Ipilimumab can cause significant autoimmune side effects. Tremelimumab (in phase III trials for melanoma) could have similar efficacy with reduced toxicities.

Anti-PD-L1: Various clinical and preclinical studies support PD-L1 as a cancer treatment target[37]. BMS-936559 is a fully human IgG4 monoclonal antibody that blocks PD-L1 from binding its two known receptors PD-1 and CD80 (http://www.onclive.com/webexclusives/the-role-of-anti-pd-11-immunotherapy-in-cancer/6\#sthash.NSf1zUJC.dpuf). It was safe in a phase I trial that included 17 OC patients $^{[37]}$ in doses of $0.3-10 \mathrm{mg} / \mathrm{kg}$ by intravenous infusion. Adverse events of any grade were reported in $91 \%$ of 207 patients. Only 12 patients $(6 \%)$ discontinued therapy for treatment-related adverse events. Common side effects included fatigue, infusion reactions, diarrhea, arthralgia, pruritis, rash, nausea, and headache. Potential immune adverse events (rash, hypothyroidism, hepatitis, sarcoidosis, diabetes mellitus, endophthalmitis, myasthenia gravis) were observed in 81 patients (39\%). Only OC patients at the $10 \mathrm{mg} / \mathrm{kg}$ dose achieved objective responses: 1 (6\%) with a partial response and $3(18 \%)$ with stable disease lasting $\geq 24$ weeks. 
Oregovomab: CA-125 is a tumor-associated antigen used to monitor OC treatment responses. CA-125 was targeted in vivo by the murine IgG1 monoclonal antibody oregovomab. Antigen-antibody complexes prime dendritic cells ${ }^{[38]}$ to activate $\mathrm{T}$ cells ${ }^{[39]}$. In a pivotal phase III study of $373 \mathrm{OC}$ patients ${ }^{[40]}$, oregovomab maintenance was used after front-line therapy. No difference in clinical outcome was identified, although treatment was well tolerated. The future for this monoclonal antibody was uncertain although interest remained. It is currently in a phase II randomized study (NCT01616303) in combination with first-line chemotherapy consisting of carboplatin plus paclitaxel versus carboplatin plus paclitaxel alone in advanced OC. As prior work suggested immune boosting effects ${ }^{[38-40]}$, this trial will study anti-CA-125 immunity in addition to clinical end points.

Abagovomab: Abagovomab is an anti-idiotypic CA-125 murine monoclonal antibody ${ }^{[41]}$ that induces anti-CA-125 antibodies. In a phase I trial, 42 OC patients were randomized to abagovomab vaccination with 2 or $0.2 \mathrm{mg}$ by intramuscular versus subcutaneous vaccination four times every 2 weeks, plus two additional monthly vaccinations. The most common adverse events were minor injection site pain, myalgia, and fever. No > grade 2 immunization-related toxicities were noted. Human anti-mouse antibodies were elicited in all patients in addition to anti-CA-125 antibodies, which were unrelated to vaccine dose or administration route ${ }^{[42]}$, prompting additional study. In a recently completed phase III trial of abagovomab maintenance therapy (the MIMOSA study) in 888 patients with stage III or IV OC ${ }^{[43]}$ in complete clinical remission after front line surgery plus platinum/taxane-based chemotherapy, patients were randomized to abagovomab $2 \mathrm{mg}$ or placebo every 2 weeks for 6 weeks as induction, followed by maintenance vaccinations every 4 weeks until recurrence. Patients were treated a mean of 450 days. Side effects were similar to the phase I trial. Vaccinations induced robust anti-CA-125 antibodies, but without increase in recurrence free or overall survival.

Volociximab: Volociximab is a chimeric IgG4 monoclonal antibody against AAB1, a component of a $5 \beta 1$ integrin that is anti-angiogenic ${ }^{[44]}$. A phase II study of weekly volociximab was conducted in 16 patients with platinum-resistant, advanced epithelial OC or primary peritoneal cancer ${ }^{[45]}$. Volociximab $15 \mathrm{mg} / \mathrm{kg}$ intravenously was given weekly until disease progression or treatment intolerance. One patient had stable disease at 8 weeks whereas the others progressed. Common adverse events included headache and fatigue in $75 \%$ of patients. Possible study-related serious adverse events in 3 patients were reversible posterior leukoencephalopathy syndrome, pulmonary embolism, and hyponatremia. This trial has prompted further assessments.

Amatuximab: Mesothelin is a tumor differentiation antigen over-expressed in certain cancers including those of ovary, pancreas and mesothelium ${ }^{[46]}$. MORAb-009 (amatuximab) is a chimeric anti-mesothelin monoclonal antibody that was tested in a phase I trial of 24 patients with mesothelin-expressing tumors including patients with $\mathrm{OC}^{[47]}$. Eleven subjects experienced stable disease prompting an ongoing phase II trial in mesothelioma patients.

Siltuximab: IL-6 in an important immunopathologic cytokine in distinct tumors ${ }^{[48]}$ and plays diverse immunopathogenic roles in OC ${ }^{[49,50]}$. Siltuximab is an anti-IL-6 antibody 
being tested as treatment for various carcinomas, hematologic malignancies and tumor cachexia $^{[51]}$.

Tocilizumab: Tocilizumab is a humanized anti-IL-6 receptor antibody being tested for cancer cachexia ${ }^{[52]}$ and is used to mitigate cytokine release symptoms in adoptive $\mathrm{T}$ cell therapy ${ }^{[53]}$. In vitro studies of tocilizumab have been reported with human OC cells but there are no reported clinical trials. Additional anti-IL-6 and anti-IL-6 receptor antibodies are in trials, including for cancer.

Anti-CD137: CD137 (4-1BB) is a stimulatory T cell co-receptor that enhances T cell proliferation and cytolytic activity. In mouse OC models, combining anti-CD137 plus anti$\mathrm{Tim}{ }^{[54]}$ or anti-PD-1 ${ }^{[55]}$ improved immune and clinical responses. Anti-CD137 has moved into phase I human clinical trials that include patients with $\mathrm{OC}^{[56]}$.

For additional information on antibody therapy for cancer, see a recent review ${ }^{[57]}$.

Additional approaches: We recently reported that the fusion toxin denileukin diftitox reduces regulatory $\mathrm{T}$ cells in human cancer and improves anti-tumor immunity, including $\mathrm{OC}$, and induced a significant partial response in one patient with metastatic OC in a phase I trial. We tested denileukin diftitox $12 \mu \mathrm{g} / \mathrm{kg}$ every $3-4$ weeks in a phase II trial of 28 OC patients. It was well-tolerated with no more than grade 2 toxicities (most commonly fatigue, fever, myalgias) but failed clinically ${ }^{[58]}$. Our recent pre-clinical findings that immune checkpoint blockade greatly enhances denileukin diftitox clinical efficacy, including in $\mathrm{OC}^{[59]}$ has prompted additional ongoing studies of combination strategies. In other preclinical studies we showed that anti-CD73 improves clinical effects of adoptive $\mathrm{T}$ cell transfer in $\mathrm{OC}^{[60]}$ and demonstrated that age ${ }^{[61]}$ and sex ${ }^{[62]}$ alter immunotherapy outcomes, factors generally not taken into account in immunotherapy trial design.

\section{Cytokines}

Interferon- $\alpha$ : Type I interferons (primarily interferons $\alpha, \beta$ and $\omega$ ) were originally identified as anti-viral proteins ${ }^{[63]}$. Soon after their discovery, they were found to block malignant cell proliferation. Interferon-a is the principal type I interferon tested for human anti-cancer activity. Studies have focused on high doses that directly inhibit tumor cell replication, but these high doses elicit significant toxicities that limit clinical applications ${ }^{[64]}$. Intraperitoneal interferon-a to treat OC was first assessed in the early 1980's, with only modest efficacy ${ }^{[65,66]}$. A phase II study of 14 patients showed that interferon-a could be administered intraperitoneally in combination with cis-platinum as OC salvage therapy when optimal surgical debulking was not achieved. The approach was tolerable with hints of clinical efficacy ${ }^{[67]}$. Intraperitoneal interferon- $a$ is ineffective against malignant OC ascites $^{[68]}$.

In a mouse OC model, interferon-a improved paclitaxel clinical efficacy ${ }^{[69]}$. Interferon-a upregulates OC cell human leukocyte antigen class I in vitro ${ }^{[70]}$ suggesting possible beneficial immune modulation. However, interferon-a down-regulated molecules HMFG1 and HMFG2, antigens that could be OC immune therapy targets. These results illustrate the 
concept that treatments effects can be multi-faceted, which must be taken into account when designing combination therapies.

We found that interferon- $a$ at low immune modulating doses improved the immune and clinical efficacy of denileukin diftitox used to deplete regulatory $\mathrm{T}$ cells in a mouse OC model, and in 2 of 3 OC patients with manageable toxicities ${ }^{[71]}$, prompting ongoing studies. Gene therapy with adenoviruses engineered to express interferon- $\beta$ was used in an early phase clinical trial that included two OC patients ${ }^{[72]}$. One of the two had stable disease two months after treatment ended, but both died within five months of treatment. Interferon- $\beta$ levels decreased after the second adenovirus infusion, because neutralizing anti-adenovirus antibodies developed, a well-known limitation of repeated adenovirus administrations. Nonetheless, anti-tumor antibodies were also generated. Finally, interferon-a reduces proliferation in human OC stem cells ${ }^{[73]}$, suggesting additional mechanisms of action.

Interferon- $\gamma$. Interferon- $\gamma$ was used to treat OC by $1992^{[74]}$, and by 1996 , intraperitoneal interferon- $\gamma$ elicited some encouraging preliminary results ${ }^{[75]}$. Interferon- $\gamma$ plus front line chemotherapy improved OC survival ${ }^{[76]}$. Interleukin-2 plus interferon- $\gamma$ was studied with infusion of tumor filtrating lymphocytes in OC. Interferon- $\gamma$ either alone or combined with interleukin-2 upregulated tumor cell human leukocyte antigen class I and class II expression ${ }^{[77]}$, suggesting augmented tumor immunogenicity. Of the $22 \mathrm{OC}$ patients receiving cytokine treatments, two also received tumor infiltrating lymphocyte adoptive transfer after ex vivo expansion. One of these two had disease stabilization $>6$ months. Interferon- $\gamma$ plus IL- 2 therapy activated $\mathrm{CD} 8^{+} \mathrm{T}$ cells but also induced potentially immunosuppressive IL-10 and TGF- $\beta$.

In a phase I trial, 25 potentially chemotherapy-sensitive OC patients with recurrent measurable disease got subcutaneous GM-CSF (starting at $400 \mu \mathrm{g} /$ day) for 7 days plus subcutaneous IFN- $\gamma(100 \mu \mathrm{g})$ on days 5 and 7 in attempts to boost antibody dependent cellular cytotoxicity, before and after carboplatin (AUC 5, intravenous). Blood myeloid cells activated monocytes increased but without clear effects on antibody dependent cellular cytotoxicity[78].

In mouse xenograft models, interferon- $\gamma$ treatment significantly improved survival of OC tumor-challenged mice. Carboplatin did not enhance the survival benefit of interferon- $\gamma$, whereas survival was enhanced by the matrix metalloprotease inhibitor batimastat ${ }^{[79]}$. In four human OC lines studied in vitro, interferon- $\gamma$ downregulated Her2 and impeded cell proliferation ${ }^{[80]}$. In another in vitro, study, interferon- $\gamma$ rendered OC cells more susceptible to cytotoxicity mediated by $\mathrm{CD} 8^{+} \mathrm{CA}-125$ (tumor)-specific $\mathrm{T}$ cells ${ }^{[81]}$.

Interleukin (IL)-2: IL-2, a T cell growth and activator factor, exerts modest anti-cancer activity in melanoma and renal cell carcinoma, among other cancers ${ }^{[82]}$. IL-2 at low doses was combined with retinoic acid in an $\mathrm{OC}$ trial ${ }^{[83]}$. Five-year progression-free survival and overall survival rates were $29 \%$ and $38 \%$, respectively in 65 evaluable OC patients. Immune effects included decreased vascular endothelial growth factor and statistically significant increases in lymphocytes and natural killer cells. In a phase II trial of $31 \mathrm{OC}$ patients with platinum-resistant or platinum-refractory disease ${ }^{[84]}$, intraperitoneal IL-2 elicited hints of 
clinical efficacy in addition to being relatively well tolerated. In 24 patients so assessed, there were four complete responses and two partial responses. Survival was positively correlated with total and interferon- $\gamma^{+} \mathrm{CD} 8^{+} \mathrm{T}$ cell numbers

IL-2 plus erythropoietin was tested in peripheral blood stem cell transplants for breast cancer and OC. Myeloid cell recovery was improved but there were no significant immune benefits ${ }^{[85]}$. Therapeutic IL-2 infusions modulate Treg numbers and trafficking in OC ${ }^{[86]}$, but the clinical significance is uncertain. However, because IL-2 is a Treg growth and differentiation factor, combining IL-2 with Treg depletion could be useful.

Tumor necrosis factor (TNF)- $\alpha$ : TNF-a can directly induce apoptosis of cancer cells and promote anticancer immune responses. TNF- $a$ fused to the tri-peptide asparagine-glycinearginine (NGR-hTNF) binds selectively to CD13, which is overexpressed on tumor blood vessels. Preclinical studies showed that NGR-hTNF exhibits higher potency than native TNF- $a$ and circumvents its toxicities. 37 patients with platinum-resistant OC were given a median of 4 cycles of NGR-hTNF ${ }^{[87]}$. Partial responses were observed in $8(23 \%)$ and stable disease in 15 (43\%). Weakness, anemia, leukopenia, nausea, neutropenia, vomiting, chills, and constipation were the most common side effects. Febrile neutropenia was observed in one patient $(3 \%)$. However, $<10 \%$ of adverse events were attributable to NGR-hTNF.

IL-18: Recombinant IL-18 (SB-485232) is an immunostimulatory cytokine that boots antitumor immunity in combination with pegylated liposomal doxorubicin in mouse models. In a phase I study, SB-485232 was combined with pegylated liposomal doxorubicin in patients with recurrent OC. 16 patients received four cycles of pegylated liposomal doxorubicin $\left(40 \mathrm{mg} / \mathrm{m}^{2}\right)$ every 28 days, plus dose-escalated SB-485232 on days 2 and 9 of each cycle plus additional discretionary pegylated liposomal doxorubicin monotherapy. Most patients (82\%) were platinum-resistant or refractory, and heavily pre-treated. SB-485232 up to $100 \mu \mathrm{g} / \mathrm{kg}$ was well-tolerated. Pegylated liposomal doxorubicin did not alter SB-485232 biologic activity and SB-485232 did not affect doxorubicin toxicities. Ten of 16 subjects $(63 \%)$ completed study and five $(31 \%)$ progressed on treatment. $6 \%$ had a partial response and $38 \%$ had stable disease ${ }^{[88]}$.

A summary of recent clinical trials using antibodies, immunotoxins, or cytokines is summarized in Table I.

\section{Other treatments}

Peptide vaccines: Many OC patients have easily detectable numbers of functional tumor antigen specific $\mathrm{T}$ cells, suggesting that augmenting tumor-specific immunity could lead to improved clinical benefits. A number of tumor-associated antigens have been detected in OC, any of which potentially could help elicit beneficial anti-tumor- immunity. These tumor-associated antigens include HER2/neu ${ }^{[5]}$, MUC1 ${ }^{[10]}$, NY-ESO-1 ${ }^{[11]}$, membrane folate receptor ${ }^{[12]}$, folate binding protein $(\mathrm{gp} 38)^{[13]}$, TAG-72 ${ }^{[14]}$, mesothelin ${ }^{[15,16]}$, sialyl$\mathrm{Tn}^{[17,18]}$, milk fat globulin-1 ${ }^{[21]}$ and $\mathrm{OA} 3^{[19]}$.

Peptide vaccines help to define the magnitude and kinetics of specific immune responses, but are limited clinically in that they are generally recognized by a single major 
histocompatibility complex molecule as they are relatively short in length. Peptide library vaccines could help overcome this shortcoming ${ }^{[89]}$ but have not specifically been tested in OC to our knowledge.

NY-ESO-1: NY-ESO-1 is highly expressed in OC. It was expressed in vaccinia or fowlpox viruses and tested in 22 patients with advanced $\mathrm{OC}$ in clinical remission ${ }^{[90]}$. Patients were given one intradermal dose of NY-ESO-1-vaccinia vector followed by monthly subcutaneous NY-ESO-1-fowlpox vector. Vaccination increased NY-ESO-1 specific antibodies, or $\mathrm{CD}^{+}$or $\mathrm{CD}^{+} \mathrm{T}$ cells. The median duration of progression-free survival was 21 months and median overall survival was 4 years. No adverse events higher than grade 2 were observed and the most common side effect was injection site pain.

A phase I trial used decitabine as an epigenetic modifier for NY-ESO-1 vaccine and liposomal doxorubicin liposome in 12 patients with relapsed OC. The regimen was safe with manageable toxicities. Vaccination increased NY-ESO-1-specific antibodies and T cells and antibodies to additional tumor antigens were elicited. Stable disease or partial clinical response was noted in $6 / 10$ evaluable patients ${ }^{[91]}$ prompting additional studies.

P53: p53 overexpression is common in many distinct cancers, including OC. Vaccination with $\mathrm{p} 53$ peptide plus IL-2, GM-CSF and montanide adjuvant was tested in patients with stage III, IV or recurrent p53-overexpressing OC without evidence of disease at vaccination. Subcutaneous vaccination improved anti-p53 immunity (interferon- $\gamma$ production and p53containing MHC tetramers) in nine of thirteen patients ${ }^{[2]}$. Subcutaneous vaccination was compared to intravenous infusion of p53-pulsed dendritic cells using IL-2 as an adjuvant/T cell enhancer. Both strategies elicited comparable immunity ${ }^{[92]}$. Thus, the logistically simpler subcutaneous approach could be the best path forward, according to study investigators. OC recurrence and survival data were not reported. IL-2 administration increased blood Treg numbers significantly, which could impede anticancer immunity, an issue that requires further investigation. Another phase II trial tested a synthetic long p53 peptide in patients with recurrent $\mathrm{OC}$ and found that it induced antigen-specific $\mathrm{T}$ cells, but did not improve clinical outcomes as a stand-alone approach, or when tested with secondary chemotherapy ${ }^{[93]}$.

Natural cancer peptides: DPX-0907 (DepoVax) is an oil-based peptide adjuvant. In a phase I trial of patients with advanced-stage cancers of breast, ovary or prostate, a vaccine of DPX-0907 plus naturally occurring HLA A2-expressed cancer peptides derived from cell lines was well-tolerated and immunogenic ${ }^{[94]}$. Injection site reactions were the most common adverse event. Vaccination induced polyfunctional $\mathrm{T}$ cells, including in OC patients, prompting additional studies.

Carcinoembryonic antigen glypican-3 (GPC3): A phase II trial tested a GPC3-derived peptide vaccine in incomplete Freund's adjuvant. OC patients received vaccination biweekly for 6 injections and then every 6 weeks until disease progression. Two OC patients with chemotherapy-refractory disease achieved partial clinical responses in this ongoing trial ${ }^{[95]}$. 
Carcinoembryonic antigen (CEA) and MUC1: CEA and MUC-1 are overexpressed many carcinomas. 25 patients were primed with a vaccinia virus expressing CEA and MUC-1 plus the costimulatory molecules CD80, intercellular adhesion molecule 1 , and lymphocyte function-associated antigen 3, PANVAC-V) and boosted with fowlpox expressing these molecules (PANVAC-F). Vaccination was well tolerated with no grade 2 toxicity in more than $2 \%$ of the cycles, except local vaccine reactions. MUC-1 and/or CEA-specific immunity was generated in 9 of 16 patients. One patient with clear cell OC had a durable (18-month) clinical response ${ }^{[96]}$.

In a follow-up study ${ }^{[97]}, 26$ patients were vaccinated with PANVAC monthly. Side effects were largely injection-site reactions. Of the $14 \mathrm{OC}$ patients, median time to progression was 2.0 months (range 1-6) and median overall survival was 15.0 months. Patients with limited tumor burden with minimal prior chemotherapy seemed to derive the most benefit from the vaccine. An OC patient from the prior trial cited above ${ }^{[96]}$ progressed after 38 months.

Additional studies are underway.

\section{Adoptive cell transfers}

Dendritic cells $(\boldsymbol{D C})$ : The role of DC in cancer therapy has been reviewed ${ }^{[98]}$. Adoptive transfer of tumor antigen-pulsed DC increases antitumor immunity by activating anti- tumor T cells. In a phase I/II trial, 11 advanced-stage OC patients received DC loaded with Her2/ neu, telomerase, and pan $T$ helper cell stimulating (PADRE) peptides \pm low dose cyclophosphamide to deplete Tregs ${ }^{[99]}$. Cell infusions were well tolerated and the most common side effects were low grade hypersensitivity reactions with no treatment-related grade 3 events. Only modest immunity was elicited by the vaccine (antigen-specific T cell cytokines or tetramer labeling). However, of 11 patients, only 1 died within 3 years of vaccination. Of the remaining 10,3 experienced chemotherapy-responsive recurrences and the rest remained disease-free. Another recent trial used autologous whole tumor lysatepulsed DC plus bevacizumab, cyclophosphamide, and autologous tumor lysate-primed T cells in recurrent OC patients ${ }^{[100]}$. Transfusions were well tolerated with no grade 3 or higher events. 2 of 6 patients experienced partial responses, and 2 exhibited stable disease. There were reduced circulating Tregs and increased tumor-specific $\mathrm{T}$ cells at study end in the 4 patients that experienced clinical benefit.

Very recently, a phase II trial of 10 OC patients with minimal residual disease tested subcutaneous autologous DC pulsed with tumor lysate and keyhole limpet hemocyanin as an adjuvant plus adjuvant low-dose IL-2 ${ }^{[101]} .3$ of 10 patients maintained complete remissions for 38-83 months and a third with complete remission relapsed after 50 months. In patients that experienced clinical benefit, multiple measures of antitumor immunity increased, such as natural killer cell activity, interferon- $\gamma^{+} \mathrm{T}$ cells, $\mathrm{T}_{\mathrm{H}} 1$-stimulating IL-12, and immunosuppressive TGF- $\beta$ declined.

DC/tumor cell fusions: Reinfusion of autologous DC fused to OC cells could induce more efficient presentation of the wide array of tumor antigens versus tumor alone. DC/tumor cell fusion has been tested in various preclinical models ${ }^{[102,103]}$, but not in human OC trials. 
T cells: The goal of adoptive $\mathrm{T}$ cell transfer in cancer immunotherapy is to increase numbers of activated, cancer-specific cytotoxic or helper $\mathrm{T}$ cells. Recent technologies have been reviewed $^{[104]}$. In a pilot study, 7 subjects with recurrent local OC were given multiple cycles of intraperitoneal infusions of autologous MUC1 peptide-stimulated cytotoxic T lymphocytes ${ }^{[105]}$. Infusions were well tolerated, multiple infusions did not offer greater benefit over one, and clinical benefit was seen in only one patient who was disease free $>12$ years.

Most recent adoptive $\mathrm{T}$ cell transfers use $\mathrm{T}$ cell receptor (TCR) transgenic or chimeric antigen receptor (CAR) T cells. Recombinant TCRs give a T cell fixed MHC-dependent specificity. CAR T cells express tumor-antigen specific antibody fragments on their surface, fused to intracellular activation proteins (e.g., $\mathrm{CD} 3 \zeta, 4-1 \mathrm{BB}, \mathrm{OX} 40)$ and recognize antigen independent of MHC. A preclinical study showed NKG2D-specific CAR T cells provide protection and establish memory against distinct $\mathrm{OC}$ tumors where only $7 \%$ cells express NKG2D ${ }^{[106]}$. Despite inducing complete remissions in leukemia patients, the efficacy of CAR T cells in solid tumors has been more limited due to inefficient tumor homing.

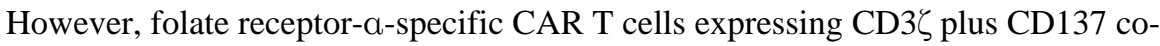
stimulatory domains protected against established OC in immunodeficient mice, underscoring the importance of the intracellular activation proteins. A phase 1 trial of OC

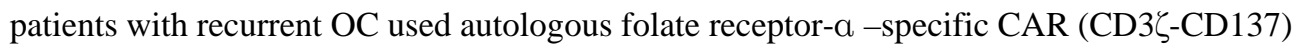
$\mathrm{T}$ cells is planned ${ }^{[107]}$.

Oncolytic viruses: Myxoma virus is non-pathogenic in humans but infects human cancer cells and exhibits oncolytic activity in preclinical models, reviewed elsewhere ${ }^{[108]}$. Myxoma virus possesses oncolytic activity against ascites-derived human OC cells in vitro ${ }^{[109]}$. However, there are currently no reported OC clinical trials with myxoma virus. Reovirus is also oncolytic against human OC cells in vitro ${ }^{[110]}$. Neutralizing antibodies in malignant ascites can inactivate reovirus oncolytic activity, which can be overcome by loading reovirus onto immature DCs or lymphokine-activated killer cells ${ }^{[111]}$. A phase I trial of reovirus in platinum-resistant OC patients is ongoing (NCT00602277).

A summary of recent clinical trials using vaccines, adoptive cell transfers, and oncolytic viruses is summarized in Table II.

\section{Conclusion}

Recent advances in understanding cancer immunotherapy and in developing novel agents has led to significant improvements in immunotherapy, most notably in malignant melanoma, but also in other cancers. There is currently no FDA-approved immunotherapy for $\mathrm{OC}$, but there is much promise from leads developed in ongoing trials in $\mathrm{OC}$ and other cancers. Over the next several years we expect that important advances in OC immunotherapy will be made, leading to important phase II and III trials. Because of a lack of curative salvage treatment options for relapsed or refractory OC, clinicians should consider referrals to early phase clinical trials, including OC immunotherapy trials. 


\section{References and Recommended Reading}

Papers of particular interest, published recently, have been highlighted as:

- Of importance

•• Of major importance

1•. Erickson BK, Conner MG, Landen CN Jr. The role of the fallopian tube in the origin of ovarian cancer. Am J Obstet Gynecol. 2013; 209(5):409-14. This paper examines evidence for the cell of origin of epithelial ovarian carcinomas. [PubMed: 23583217]

2. Ioannides CG, et al. Cytotoxic T-cell clones isolated from ovarian tumour infiltrating lymphocytes recognize common determinants on non-ovarian tumour clones. Scand J Immunol. 1993; 37(4): 413-24. [PubMed: 8469924]

3. Peoples GE, et al. Breast and ovarian cancer-specific cytotoxic $\mathrm{T}$ lymphocytes recognize the same HER2/neu-derived peptide. Proc Natl Acad Sci U S A. 1995; 92(2):432-6. [PubMed: 7831305]

4. Wagner U, et al. Immunological responses to the tumor-associated antigen CA125 in patients with advanced ovarian cancer induced by the murine monoclonal anti-idiotype vaccine ACA125. Hybridoma. 1997; 16(1):33-40. [PubMed: 9085126]

5. Disis ML, et al. Generation of T-cell immunity to the HER-2/neu protein after active immunization with HER-2/neu peptide-based vaccines. J Clin Oncol. 2002; 20(11):2624-32. [PubMed: 12039923]

6. Knutson KL, et al. Immunization of cancer patients with a HER-2/neu, HLA-A2 peptide, p369-377, results in short-lived peptide-specific immunity. Clin Cancer Res. 2002; 8(5):1014-8. [PubMed: 12006513]

7. Qian HN, et al. The experimental study of ovarian carcinoma vaccine modified by human B7-1 and IFN-gamma genes. Int J Gynecol Cancer. 2002; 12(1):80-5. [PubMed: 11860540]

8. Curiel TJ, et al. Blockade of B7-H1 improves myeloid dendritic cell-mediated antitumor immunity. Nat Med. 2003; 9(5):562-7. [PubMed: 12704383]

9••. Curiel TJ, et al. Specific recruitment of regulatory $\mathrm{T}$ cells in ovarian carcinoma fosters immune privilege and predicts reduced survival. Nat Med. 2004; 10(9):942-9. This paper shows for the first time that regulatory $\mathrm{T}$ cells in the tumor (as opposed to those from blood) are immunopathologic and correlate negatively with survival. This is the first demonstration that low numbers of intratumor regulatory $\mathrm{T}$ cells defeat otherwise beneficial anti-cancer immunity and establishes the rationale to deplete regulatory $\mathrm{T}$ cells as ovarian cancer immunotherapy. [PubMed: 15322536]

10. Vlad AM, et al. MUC1 immunobiology: from discovery to clinical applications. Adv Immunol. 2004; 82:249-93. [PubMed: 14975259]

11. Diefenbach CS, et al. Safety and immunogenicity study of NY-ESO-1b peptide and montanide ISA-51 vaccination of patients with epithelial ovarian cancer in high-risk first remission. Clin Cancer Res. 2008; 14(9):2740-8. [PubMed: 18451240]

12. Coliva A, et al. 90Y Labeling of monoclonal antibody MOv18 and preclinical validation for radioimmunotherapy of human ovarian carcinomas. Cancer Immunol Immunother. 2005; 54(12): 1200-13. [PubMed: 15926078]

13. Mantovani LT, et al. Folate binding protein distribution in normal tissues and biological fluids from ovarian carcinoma patients as detected by the monoclonal antibodies MOv18 and MOv19. Eur J Cancer. 1994; 30A(3):363-9. [PubMed: 8204360]

14. Thor A, et al. Tumor-associated glycoprotein (TAG-72) in ovarian carcinomas defined by monoclonal antibody B72.3. J Natl Cancer Inst. 1986; 76(6):995-1006. [PubMed: 3520078]

15. Chang K, Pastan I. Molecular cloning of mesothelin, a differentiation antigen present on mesothelium, mesotheliomas, and ovarian cancers. Proc Natl Acad Sci U S A. 1996; 93(1):13640. [PubMed: 8552591]

16. Cheng WF, et al. High mesothelin correlates with chemoresistance and poor survival in epithelial ovarian carcinoma. Br J Cancer. 2009; 100(7):1144-53. [PubMed: 19293794] 
17. Inoue $\mathrm{M}$, et al. Clinical value of sialyl Tn antigen in patients with gynecologic tumors. Obstet Gynecol. 1990; 75(6):1032-6. [PubMed: 2342728]

18. Sandmaier BM, et al. Evidence of a cellular immune response against sialyl-Tn in breast and ovarian cancer patients after high-dose chemotherapy, stem cell rescue, and immunization with Theratope STn-KLH cancer vaccine. J Immunother. 1999; 22(1):54-66. [PubMed: 9924700]

19. Campbell IG, et al. An ovarian tumor marker with homology to vaccinia virus contains an IgV-like region and multiple transmembrane domains. Cancer Res. 1992; 52(19):5416-20. [PubMed: 1394148]

20••. Zhang L, et al. Intratumoral T cells, recurrence, and survival in epithelial ovarian cancer. N Engl J Med. 2003; 348(3):203-13. This landmark paper shows for the first time that endogenous host immunity is important to protection from ovarian cancer. This finding provides a strong rationale for immunotherapy to treat ovarian cancer. [PubMed: 12529460]

21. Epenetos AA, et al. Antibody-guided irradiation of advanced ovarian cancer with intraperitoneally administered radiolabeled monoclonal antibodies. J Clin Oncol. 1987; 5(12):1890-9. [PubMed: 3681374]

22. Kalbasi AJC, Haas N, Vapiwala N. Radiation and immunotherapy: a synergistic ccombination. Journal of Clin Invest. 2013; 123(7):2756-63.

23. Bookman MA. First-line chemotherapy in epithelial ovarian cancer. Clin Obstet Gynecol. 2012; 55(1):96-113. This paper is a useful introduction to approved agents to treat epithelial ovarian cancer. [PubMed: 22343232]

24. Beyer M, et al. Reduced frequencies and suppressive function of CD4+CD25hi regulatory T cells in patients with chronic lymphocytic leukemia after therapy with fludarabine. Blood. 2005; 106(6): 2018-25. [PubMed: 15914560]

25. Motoyoshi Y, et al. Different mechanisms for anti-tumor effects of low- and high-dose cyclophosphamide. Oncol Rep. 2006; 16(1):141-6. [PubMed: 16786137]

26. Vincent J, et al. 5-Fluorouracil selectively kills tumor-associated myeloid-derived suppressor cells resulting in enhanced T cell-dependent antitumor immunity. Cancer Res. 2010; 70(8):3052-61. [PubMed: 20388795]

27•. Apetoh L, et al. Immunogenicity of anthracyclines: moving towards more personalized medicine. Trends Mol Med. 2008; 14(4):141-51. This paper is a good overview of how traditional cytotoxic agents could be used to improve cancer immunotherapy. [PubMed: 18353726]

28. Riva P, et al. Locoregional immunotherapy of human ovarian cancer: preliminary results. Int J Rad Appl Instrum B. 1989; 16(6):659-66. [PubMed: 2606721]

29. Stewart JS, et al. Intraperitoneal yttrium-90-labeled monoclonal antibody in ovarian cancer. J Clin Oncol. 1990; 8(12):1941-50. [PubMed: 2230887]

30. Hird V, et al. Adjuvant therapy of ovarian cancer with radioactive monoclonal antibody. $\mathrm{Br} \mathrm{J}$ Cancer. 1993; 68(2):403-6. [PubMed: 8347497]

31. Epenetos AA, et al. Long term survival of patients with advanced ovarian cancer treated with intraperitoneal radioimmunotherapy. Int J Gynecol Cancer. 2000; 10(S1):44-46. [PubMed: 11240732]

32. Oei AL, et al. Decreased intraperitoneal disease recurrence in epithelial ovarian cancer patients receiving intraperitoneal consolidation treatment with yttrium-90-labeled murine HMFG1 without improvement in overall survival. Int J Cancer. 2007; 120(12):2710-4. [PubMed: 17354223]

33. Armstrong DK, et al. Farletuzumab (a monoclonal antibody against folate receptor alpha) in relapsed platinum-sensitive ovarian cancer. Gynecol Oncol. 2013; 129(3):452-8. [PubMed: 23474348]

34. Konner JA, et al. Farletuzumab, a humanized monoclonal antibody against folate receptor alpha, in epithelial ovarian cancer: a phase I study. Clin Cancer Res. 2010; 16(21):5288-95. [PubMed: 20855460]

35. Sehouli J, et al. Catumaxomab with and without prednisolone premedication for the treatment of malignant ascites due to epithelial cancer: results of the randomised phase IIIb CASIMAS study. Med Oncol. 2014; 31(8):76. [PubMed: 24965536] 
36••. Pardoll DM. The blockade of immune checkpoints in cancer immunotherapy. Nat Rev Cancer. 2012; 12(4):252-64. This is an excellent review of immune checkpoint blockade, a promising approach to cancer immunotherapy. [PubMed: 22437870]

37. Brahmer JR, et al. Safety and activity of anti-PD-L1 antibody in patients with advanced cancer. N Engl J Med. 2012; 366(26):2455-65. [PubMed: 22658128]

38. Berek JS. Immunotherapy of ovarian cancer with antibodies: a focus on oregovomab. Expert Opin Biol Ther. 2004; 4(7):1159-65. [PubMed: 15268682]

39. Ehlen TG, et al. A pilot phase 2 study of oregovomab murine monoclonal antibody to CA125 as an immunotherapeutic agent for recurrent ovarian cancer. Int J Gynecol Cancer. 2005; 15(6):102334. [PubMed: 16343178]

40. Berek J, et al. Oregovomab maintenance monoimmunotherapy does not improve outcomes in advanced ovarian cancer. J Clin Oncol. 2009; 27(3):418-25. [PubMed: 19075271]

41. Pfisterer J, et al. Abagovomab for ovarian cancer. Expert Opin Biol Ther. 2011; 11(3):395-403. [PubMed: 21241213]

42. Sabbatini $\mathrm{P}$, et al. Phase I study of abagovomab in patients with epithelial ovarian, fallopian tube, or primary peritoneal cancer. Clin Cancer Res. 2006; 12(18):5503-10. [PubMed: 17000686]

43. Sabbatini P, et al. Abagovomab as maintenance therapy in patients with epithelial ovarian cancer: a phase III trial of the AGO OVAR, COGI, GINECO, and GEICO--the MIMOSA study. J Clin Oncol. 2013; 31(12):1554-61. [PubMed: 23478059]

44. Almokadem S, Belani CP. Volociximab in cancer. Expert Opin Biol Ther. 2012; 12(2):251-7. [PubMed: 22192080]

45. Bell-McGuinn KM, et al. A phase II, single-arm study of the anti-alpha5beta1 integrin antibody volociximab as monotherapy in patients with platinum-resistant advanced epithelial ovarian or primary peritoneal cancer. Gynecol Oncol. 2011; 121(2):273-9. [PubMed: 21276608]

46. Hassan R, Bera T, Pastan I. Mesothelin: a new target for immunotherapy. Clin Cancer Res. 2004; 10(12 Pt 1):3937-42. [PubMed: 15217923]

47. Hassan R, et al. Phase I clinical trial of the chimeric anti-mesothelin monoclonal antibody MORAb-009 in patients with mesothelin-expressing cancers. Clin Cancer Res. 2010; 16(24): 6132-8. [PubMed: 21037025]

48. Taniguchi K, Karin M. IL-6 and related cytokines as the critical lynchpins between inflammation and cancer. Semin Immunol. 2014; 26(1):54-74. [PubMed: 24552665]

49. Coward J, et al. Interleukin-6 as a therapeutic target in human ovarian cancer. Clin Cancer Res. 2011; 17(18):6083-96. [PubMed: 21795409]

50. Kryczek I, et al. B7-H4 expression identifies a novel suppressive macrophage population in human ovarian carcinoma. J Exp Med. 2006; 203(4):871-81. [PubMed: 16606666]

51. Middleton K, et al. Interleukin-6: an angiogenic target in solid tumours. Crit Rev Oncol Hematol. 2014; 89(1):129-39. [PubMed: 24029605]

52. Berti A, et al. Assessment of tocilizumab in the treatment of cancer cachexia. J Clin Oncol. 2013; 31(23):2970. [PubMed: 23816970]

53. Maude SL, et al. Managing cytokine release syndrome associated with novel T cell-engaging therapies. Cancer J. 2014; 20(2):119-22. [PubMed: 24667956]

54- Guo Z, et al. Combined TIM-3 blockade and CD137 activation affords the long-term protection in a murine model of ovarian cancer. J Transl Med. 2013; 11:215. This paper demonstrates in a preclinical model how combination immunotherapy could be better than individual immunotherapy agents to treat ovarian cancer. [PubMed: 24044888]

55. Wei H, et al. Combinatorial PD-1 blockade and CD137 activation has therapeutic efficacy in murine cancer models and synergizes with cisplatin. PLoS One. 2013; 8(12):e84927. This paper demonstrates in a pre-clinical model how combination immunotherapy could be better than individual immunotherapy agents to treat ovarian cancer, and how immunotherapy can be combined with a standard cytotoxic agent. [PubMed: 24367702]

56. Vinay DS, Kwon BS. Immunotherapy of cancer with 4-1BB. Mol Cancer Ther. 2012; 11(5):106270. [PubMed: 22532596]

Curr Treat Options Oncol. Author manuscript; available in PMC 2016 January 01. 
57••. Scott AM, Wolchok JD, Old LJ. Antibody therapy of cancer. Nat Rev Cancer. 2012; 12(4):27887. This review thoroughly covers the use of antibodies in cancer immunotherapy. [PubMed: 22437872]

58• Thibodeaux, SR., et al. Denileukin diftitox depletes regulatory T cells without clinical benefit in advanced stage epithelial ovarian carcinoma. American Association of Immunologists 2014 meeting; 2014. p. abstract 1944021 This is the first report showing that regulatory T cell depletion is possible in human ovarian cancer, with a positive clinical response in one patient, and positive immune responses in others. However, the single-agent phase II trial in ovarian cancer failed to establish significant clinical efficacy. The related manuscript has been submitted for publication

59. Murthy, K., et al. B7-H1 blockade improves efficacy of regulatory T cell depletion as cancer immunotherapy by reducing Treg regeneration, possibly through tumor B7-H1 effects. American Association of Immunologists 2014 meeting; 2014; p. abstract number 1944036

60. Jin D, et al. CD73 on tumor cells impairs antitumor T-cell responses: a novel mechanism of tumorinduced immune suppression. Cancer Res. 2010; 70(6):2245-55. [PubMed: 20179192]

61. Hurez V, et al. Mitigating age-related immune dysfunction heightens the efficacy of tumor immunotherapy in aged mice. Cancer Res. 2012; 72(8):2089-99. [PubMed: 22496463]

62. Lin PY, et al. B7-H1-Dependent Sex-Related Differences in Tumor Immunity and Immunotherapy Responses. J Immunol. 2010; 185(5):2747-2753. [PubMed: 20686128]

63. Pestka S, Krause CD, Walter MR. Interferons, interferon-like cytokines, and their receptors. Immunol Rev. 2004; 202:8-32. [PubMed: 15546383]

64. Kirkwood J. Cancer immunotherapy: the interferon-alpha experience. Semin Oncol. 2002; 29(3 Suppl 7):18-26. [PubMed: 12068384]

65. Berek JS, et al. Intraperitoneal recombinant alpha-interferon for "salvage" immunotherapy in stage III epithelial ovarian cancer: a Gynecologic Oncology Group Study. Cancer Res. 1985; 45(9): 4447-53. [PubMed: 4028027]

66. Bezwoda WR, Seymour L, Dansey R. Intraperitoneal recombinant interferon-alpha $2 \mathrm{~b}$ for recurrent malignant ascites due to ovarian cancer. Cancer. 1989; 64(5):1029-33. [PubMed: 2758381]

67. Nardi M, et al. Intraperitoneal recombinant alpha-2-interferon alternating with cisplatin as salvage therapy for minimal residual-disease ovarian cancer: a phase II study. J Clin Oncol. 1990; 8(6): 1036-41. [PubMed: 2189953]

68. Stuart GC, et al. Intraperitoneal interferon in the management of malignant ascites. Cancer. 1993; 71(6):2027-30. [PubMed: 7680276]

69. Tedjarati S, et al. Synergistic therapy of human ovarian carcinoma implanted orthotopically in nude mice by optimal biological dose of pegylated interferon alpha combined with paclitaxel. Clin Cancer Res. 2002; 8(7):2413-22. [PubMed: 12114447]

70. Metcalf KS, et al. Culture of ascitic ovarian cancer cells as a clinically-relevant ex vivo model for the assessment of biological therapies. Eur J Gynaecol Oncol. 1998; 19(2):113-9. [PubMed: 9611047]

71 . Thibodeaux, SR., et al. Interferon-a augments the clinical efficacy of regulatory $\mathrm{T}$ cell depletion in ovarian cancer through direct and indirect T cell effects; American Association of Immunologists Annual Meeting; Pittsburgh, PA. 2014; p. abstract number 1943855This is the first report showing that combination immunotherapy could improve clinical outcomes in ovarian cancer in human patients. The related manuscript has been submitted for publication

72. Sterman DH, et al. A phase I trial of repeated intrapleural adenoviral-mediated interferon-beta gene transfer for mesothelioma and metastatic pleural effusions. Mol Ther. 2010; 18(4):852-60. [PubMed: 20068553]

73. Moserle L, et al. The side population of ovarian cancer cells is a primary target of IFN-alpha antitumor effects. Cancer Res. 2008; 68(14):5658-68. [PubMed: 18632618]

74. Chen JT, Hasumi K, Masubuchi K. Interferon-alpha, interferon-gamma and sizofiran in the adjuvant therapy in ovarian cancer--a preliminary trial. Biotherapy. 1992; 5(4):275-80. [PubMed: 1290723]

75. Pujade-Lauraine E, et al. Intraperitoneal recombinant interferon gamma in ovarian cancer patients with residual disease at second-look laparotomy. J Clin Oncol. 1996; 14(2):343-50. [PubMed: 8636742] 
76. Windbichler $\mathrm{GH}$, et al. Interferon-gamma in the first-line therapy of ovarian cancer: a randomized phase III trial. Br J Cancer. 2000; 82(6):1138-44. [PubMed: 10735496]

77. Freedman RS, et al. Clinical and biological effects of intraperitoneal injections of recombinant interferon-gamma and recombinant interleukin 2 with or without tumor-infiltrating lymphocytes in patients with ovarian or peritoneal carcinoma. Clin Cancer Res. 2000; 6(6):2268-78. [PubMed: 10873077]

78. Apte SM, et al. Cytokines, GM-CSF and IFNgamma administered by priming and postchemotherapy cycling in recurrent ovarian cancer patients receiving carboplatin. J Transl Med. 2006; 4:16. [PubMed: 16603073]

79. Burke F, et al. Interferon gamma induces cell cycle arrest and apoptosis in a model of ovarian cancer: enhancement of effect by batimastat. Eur J Cancer. 1997; 33(7):1114-21. [PubMed: 9376192]

80. Marth C, et al. Gamma-interferon reduces expression of the protooncogene c-erbB-2 in human ovarian carcinoma cells. Cancer Res. 1990; 50(21):7037-41. [PubMed: 2119884]

81. Madiyalakan R, et al. OVAREX MAb-B43.13:IFN-gamma could improve the ovarian tumor cell sensitivity to CA125-specific allogenic cytotoxic T cells. Hybridoma. 1997; 16(1):41-5. [PubMed: 9085127]

82. Antony GK, Dudek AZ. Interleukin 2 in cancer therapy. Curr Med Chem. 17(29):3297-302. [PubMed: 20712575]

83. Recchia F, et al. Maintenance immunotherapy in recurrent ovarian cancer: long term follow-up of a phase II study. Gynecol Oncol. 116(2):202-7. [PubMed: 19880164]

84. Vlad AM, et al. A phase II trial of intraperitoneal interleukin-2 in patients with platinum-resistant or platinum-refractory ovarian cancer. Cancer Immunol Immunother. 2009

85. Perillo A, et al. Administration of low-dose interleukin-2 plus G-CSF/EPO early after autologous PBSC transplantation: effects on immune recovery and NK activity in a prospective study in women with breast and ovarian cancer. Bone Marrow Transplant. 2002; 30(9):571-8. [PubMed: 12407431]

86. Wei S, et al. Interleukin-2 administration alters the CD4+FOXP3+ T-cell pool and tumor trafficking in patients with ovarian carcinoma. Cancer Res. 2007; 67(15):7487-94. [PubMed: 17671219]

87. Lorusso D, et al. Phase II study of NGR-hTNF in combination with doxorubicin in relapsed ovarian cancer patients. Br J Cancer. 2012; 107(1):37-42. [PubMed: 22644293]

88. Simpkins F, et al. Chemoimmunotherapy using pegylated liposomal Doxorubicin and interleukin-18 in recurrent ovarian cancer: a phase I dose-escalation study. Cancer Immunol Res. 2013; 1(3):168-78. [PubMed: 24777679]

89. Mirshahidi S, et al. Overlapping synthetic peptides encoding TPD52 as breast cancer vaccine in mice: prolonged survival. Vaccine. 2009; 27(12):1825-33. [PubMed: 19201387]

90. Odunsi K, et al. Efficacy of vaccination with recombinant vaccinia and fowlpox vectors expressing NY-ESO-1 antigen in ovarian cancer and melanoma patients. Proc Natl Acad Sci U S A. 2012; 109(15):5797-802. [PubMed: 22454499]

91. Odunsi K, et al. Epigenetic potentiation of NY-ESO-1 vaccine therapy in human ovarian cancer. Cancer Immunol Res. 2014; 2(1):37-49. [PubMed: 24535937]

92. Rahma OE, et al. A gynecologic oncology group phase II trial of two p53 peptide vaccine approaches: subcutaneous injection and intravenous pulsed dendritic cells in high recurrence risk ovarian cancer patients. Cancer Immunol Immunother. 2012; 61(3):373-84. [PubMed: 21927947]

93. Leffers $\mathrm{N}$, et al. Long-term clinical and immunological effects of $\mathrm{p} 53-\mathrm{SLP}(\mathrm{R})$ vaccine in patients with ovarian cancer. Int J Cancer. 2012; 130(1):105-12. [PubMed: 21328579]

94. Karkada M, Berinstein NL, Mansour M. Therapeutic vaccines and cancer: focus on DPX-0907. Biologics. 2014; 8:27-38. [PubMed: 24596453]

95. Suzuki S, et al. Significant clinical response of progressive recurrent ovarian clear cell carcinoma to glypican-3-derived peptide vaccine therapy: two case reports. Hum Vaccin Immunother. 2014; 10(2):338-43. [PubMed: 24252799]

96. Gulley JL, et al. Pilot study of vaccination with recombinant CEA-MUC-1-TRICOM poxviralbased vaccines in patients with metastatic carcinoma. Clin Cancer Res. 2008; 14(10):3060-9. 
This report highlights important features of a pox-based vaccine strategy based on the TRICOM backbone, which is showing promise in early phase trials. [PubMed: 18483372]

97. Mohebtash M, et al. A pilot study of MUC-1/CEA/TRICOM poxviral-based vaccine in patients with metastatic breast and ovarian cancer. Clin Cancer Res. 2011; 17(22):7164-73. [PubMed: 22068656]

98. Palucka K, Banchereau J. Dendritic-cell-based therapeutic cancer vaccines. Immunity. 2013; 39(1): 38-48. [PubMed: 23890062]

99. Chu CS, et al. Phase I/II randomized trial of dendritic cell vaccination with or without cyclophosphamide for consolidation therapy of advanced ovarian cancer in first or second remission. Cancer Immunol Immunother. 2012; 61(5):629-41. [PubMed: 22021066]

100. Kandalaft LE, et al. Autologous lysate-pulsed dendritic cell vaccination followed by adoptive transfer of vaccine-primed ex vivo co-stimulated $\mathrm{T}$ cells in recurrent ovarian cancer. Oncoimmunology. 2013; 2(1):e22664. [PubMed: 23482679]

101. Baek S, et al. Therapeutic DC vaccination with IL-2 as a consolidation therapy for ovarian cancer patients: a phase I/II trial. Cell Mol Immunol. 2014

102. Gong J, et al. Fusions of human ovarian carcinoma cells with autologous or allogeneic dendritic cells induce antitumor immunity. J Immunol. 2000; 165(3):1705-11. [PubMed: 10903782]

103. Koido S, et al. Assessment of fusion cells from patient-derived ovarian carcinoma cells and dendritic cells as a vaccine for clinical use. Gynecol Oncol. 2005; 99(2):462-71. [PubMed: 16137749]

104 •. Kalos M, June CH. Adoptive T cell transfer for cancer immunotherapy in the era of synthetic biology. Immunity. 2013; 39(1):49-60. This report highlights features of adoptive CAR T cell transfer, a highly promising cancer immunotherapy approach. [PubMed: 23890063]

105. Wright SE, et al. Cytotoxic T-lymphocyte immunotherapy for ovarian cancer: a pilot study. J Immunother. 2012; 35(2):196-204. [PubMed: 22306908]

106. Spear P, et al. NKG2D CAR T-cell therapy inhibits the growth of NKG2D ligand heterogeneous tumors. Immunol Cell Biol. 2013; 91(6):435-40. [PubMed: 23628805]

107. Kandalaft LE, Powell DJ Jr, Coukos G. A phase I clinical trial of adoptive transfer of folate receptor-alpha redirected autologous T cells for recurrent ovarian cancer. J Transl Med. 2012; 10:157. [PubMed: 22863016]

108. Chan WM, Rahman MM, McFadden G. Oncolytic myxoma virus: the path to clinic. Vaccine. 2013; 31(39):4252-8. [PubMed: 23726825]

109. Correa RJ, et al. Myxoma virus-mediated oncolysis of ascites-derived human ovarian cancer cells and spheroids is impacted by differential AKT activity. Gynecol Oncol. 2012; 125(2):441-50. [PubMed: 22306204]

110. Hirasawa K, et al. Oncolytic reovirus against ovarian and colon cancer. Cancer Res. 2002; 62(6): 1696-701. [PubMed: 11912142]

111. Jennings VA, et al. Lymphokine-activated killer and dendritic cell carriage enhances oncolytic reovirus therapy for ovarian cancer by overcoming antibody neutralization in ascites. Int $\mathrm{J}$ Cancer. 2014; 134(5):1091-101. [PubMed: 23982804] 


\section{Opinion Statement}

All work referenced herein relates to treatment of epithelial ovarian carcinomas, as their treatment differs from ovarian germ cell cancers and other rare ovarian cancers, the treatments of which are addressed elsewhere. Fallopian tube cancers and primary peritoneal adenocarcinomatosis are also generally treated as epithelial ovarian cancers. The standard of care initial treatment of advanced stage epithelial ovarian cancer is optimal debulking surgery as feasible plus chemotherapy with a platinum plus a taxane agent. If this front-line approach fails, as it too often the case, several FDA-approved agents are available for salvage therapy. However, because no second-line therapy for advanced-stage epithelial ovarian cancer is typically curative, we prefer referral to clinical trials as logistically feasible, even if it means referring patients outside our system. Immune therapy has a sound theoretical basis for treating carcinomas generally, and for treating ovarian cancer in particular. Advances in understanding the immunopathogenic basis of ovarian cancer, and the immunopathologic basis for prior failures of immunotherapy for it and other carcinomas promises to afford novel treatment approaches with potential for significant efficacy, and reduced toxicities compared to cytotoxic agents. Thus, referral to early phase immunotherapy trials for ovarian cancer patients that fail conventional treatment merits consideration. 


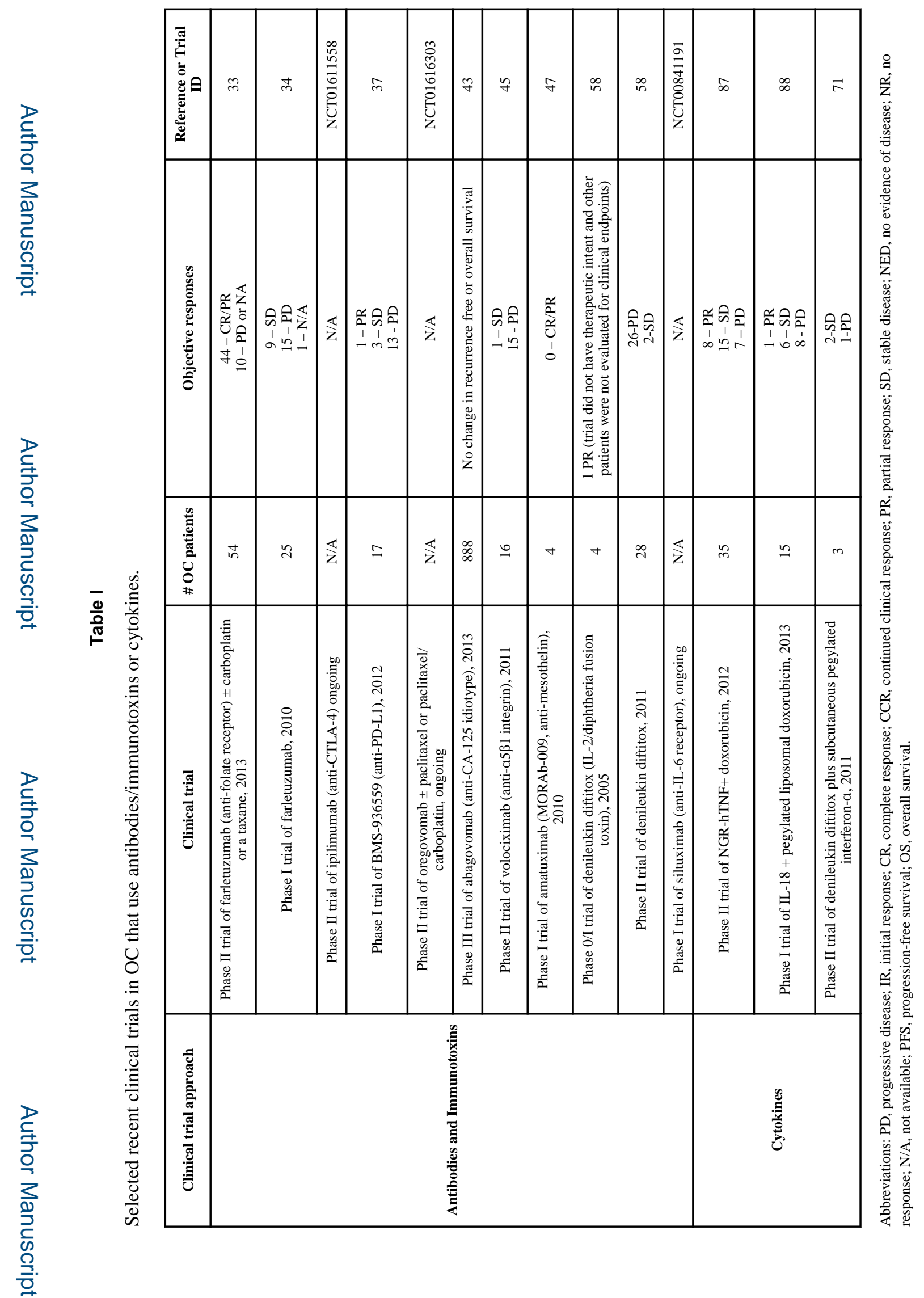

Curr Treat Options Oncol. Author manuscript; available in PMC 2016 January 01. 


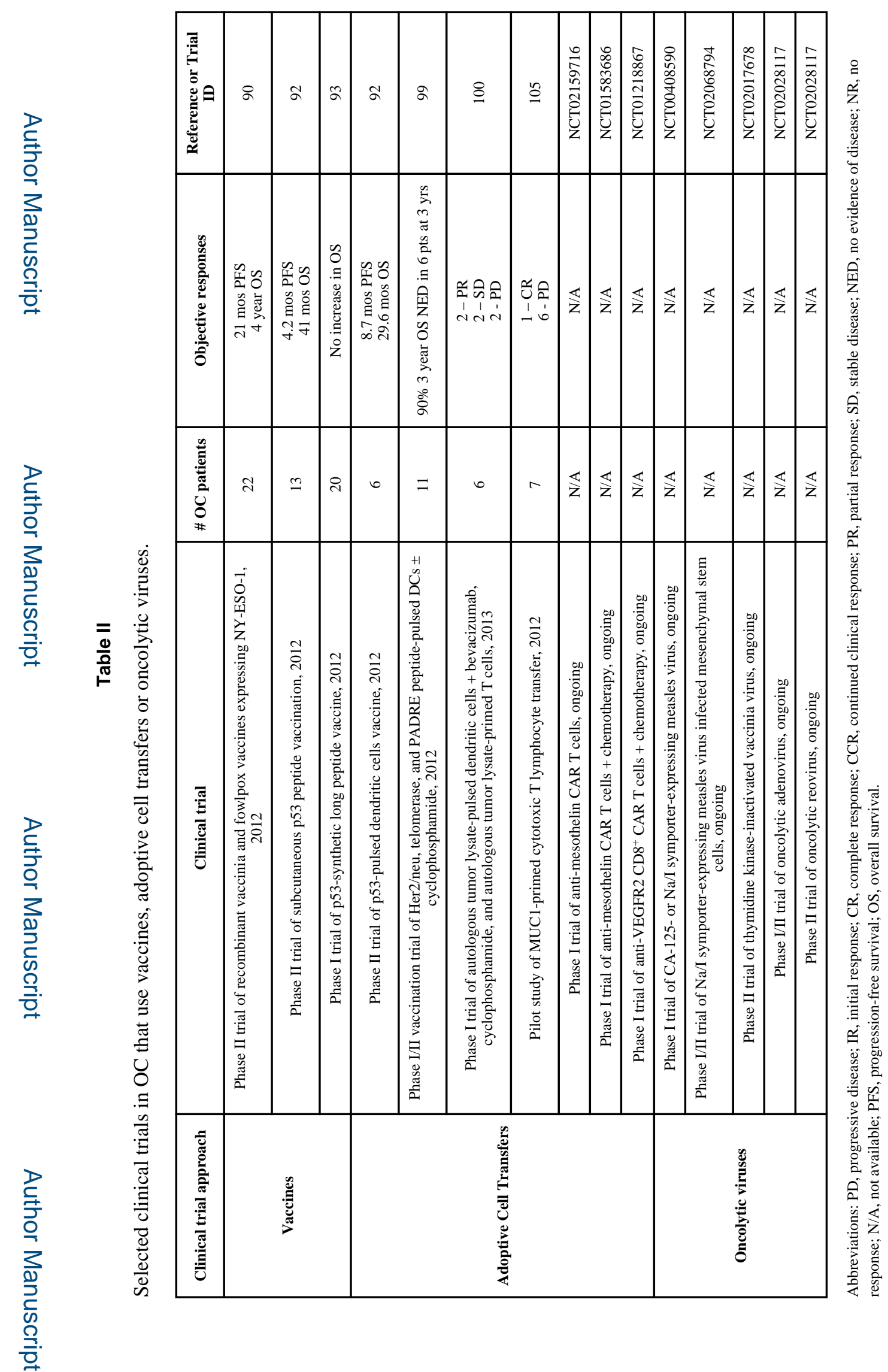

Curr Treat Options Oncol. Author manuscript; available in PMC 2016 January 01. 